\title{
Zum ägyptischen Lautwandel zwischen "" und Gutturalen
}

\author{
Stefan Bojowald \\ Universität Bonn
}

\begin{abstract}
In this article, the exchange of "c" and gutturals is investigated in detail. Research so far has dealt only with the exchange of "ल" and " $k$ ". The current contribution extends the treatment to the two other gutturals " $k$ " and " $g$ ". It will be shown that the same rule applies to all three gutturals.
\end{abstract}

Keywords: Old Egyptian language, phonetic changes/shifts in the Egyptian language, phonetic change/shift between "c" and gutturals.

Der Lautwandel zwischen „" und Gutturalen stellt in der bisherigen ägyptologischen Forschung ein Randthema dar. Die einzige Behandlung scheint er bei Westendorf ${ }^{1}$ erhalten $z u$ haben, durch den die wissenschaftliche Öffentlichkeit zum ersten Mal in größerem Ausmaß auf diesen Tatbestand aufmerksam geworden ist. In der Zeit

\footnotetext{
${ }^{1}$ W. WESTENDORF, Grammatik der medizinischen Texte, Grundriss der Medizin der alten Ägypter VIII (Berlin, 1962), 19/20.
} 
davor hatte Sethe ${ }^{2}$ lediglich auf die Umstellung von „“" und ,,$k^{\prime \prime}$ im Wort „h $\underline{c} k^{\prime \prime}$ „scheren“ verwiesen, das sich im Koptischen als „2шஸке“ erhalten hat, aber noch nicht an den Lautwandel als Ursache dafür gedacht. In der Literatur ist offenbar sonst kein Hinweis auf dieses Phänomen $\mathrm{zu}$ finden. In der vor genau fünfzig Jahren erschienenen Arbeit hatte Westendorf nur den Wechsel zwischen „““ und , $k^{\prime \prime}$ betrachtet, was zum Teil wohl an der begrenzten Textauswahl gelegen hatte. Die These hatte Westendorf auf drei sichere Beispiele stützen können. Im ersten Fall hatte er das Augenmerk auf die Schreibung , ,śr" in Sm $46(15,20)$ anstelle von , śk $k r^{\prime \prime}$ „Schlag" in Sm $46(16,13)$ gelenkt. Die Beweisführung hatte er durch die Heranziehung der Schreibung "rrf" „zusammenschliesen“ anstelle von „krf" fortgesetzt. Der Schlussstrich unter die Argumentation war mit dem Rekurs auf die Schreibung „krf“ für „ "rff“ „Beutel“ gezogen worden. Die Erscheinung hatte Westendorf außerdem aus der ,,$m n^{\top} . t^{\star{ }^{* 3}}$

\footnotetext{
${ }^{2}$ K. SETHE, Das aegyptische Verbum im Altaegyptischen, Neuaegyptischen und Koptischen, Erster Band, Laut- und Stammeslehre (Leipzig: J. C. Hinrichs'sche Buchhandlung, 1899), 90.

${ }^{3}$ Zum Wort „mn`.t" „Amme“ vgl. C. PEUST, Egyptian Phonology, An Introduction to the Phonology of a dead Language (Göttingen: Peust \& Gutschmidt Verlag, 1999), 236/238/255. Zur , $m n^{\ulcorner} \cdot t^{\prime \prime}$ - Amme im Allgemeinen und Ausländerinnen als „,mn'. $t^{\text {“ }}$ Amme im Besonderen vgl. TH. SCHNEIDER, Ausländer in Ägypten während des Mittleren Reiches und der Hyksoszeit, Teil 2, Die ausländische Bevölkerung, Ägypten und Altes Testament 42 (Wiesbaden: Harrassowitz, 2003), 285. Die „,mn $n^{\complement} . t^{4}$ - Amme hat im Übrigen zwei Wortspiele gebildet, zu denen hier kurz Stellung genommen werden soll. Der erste Hinweis gilt dem Wortspiel zwischen „mn $m$. $t^{\text {“ }}$ „Amme“ und „mni" „hüten“, das bei H.-J. THISSEN, Der demotische Ammenvertrag aus Tebtynis (Tafel 32-34), in: H.-J. THISSEN/K.-TH. ZAUZICH (Hrsg.), Grammata Demotika, Festschrift für Erich Lüddeckens zum 15. Juni 1983 (Würzburg: Gisela Zauzich Verlag, 1984), 237 3, zu finden ist. Der gleiche Sprachgebrauch ist im Koptischen verwendet worden, wo in der sahidischen Version der Pachomviten bei L. TH. LEFORT, S. Pachomii vitae, Sahidice schriptae, Corpus Scriptorum Christianorum Orientalium 99/100 (Louvain: Imprimerie Orientaliste L. Durbecq, 1952), 181, 19, das Wort „MOoNe“" „weiden“ ebenfalls für eine Amme gebraucht worden ist. Das zweite Wortspiel ist bei H. GOEDICKE/E. F. WENTE, Ostraka Michaelides (Wiesbaden: Harrassowitz, 1962), Taf. XV, zwischen „mn`.t" „Amme“ und „Imn“ „Amun“ entwickelt worden, indem es seine Kraft aus dem Lautwandel zwischen "“" und "i" bezogen hat; zu diesem Lautwandel vgl. K. SETHE, Das aegyptische Verbum im Altaegyptischen, Neuaegyptischen und Koptischen, Erster Band, Laut- und Stammeslehre (Leipzig: J. C. Hinrichs'sche Buchhandlung, 1899), 88/90; C. PEUST, Egyptian Phonology, An Introduction to the Phonology of a dead Language (Göttingen: Peust \& Gutschmidt Verlag, 1999), 103/104; W.
} 
„Amme“ erschließen können, deren Gegenstück im Hebräischen "מנקתת, gelautet hat. Der Lautwandel hat nach den Worten von Westendorf darüber hinaus die Ursache für den Wegfall von ", "nach ,$k^{“}$ in den Wörtern ,nk.w“ (Eb 296) für , $n k^{\complement} \cdot w t^{“}$ (Eb 102), „stechende Schmerzen (ebenso, ,nk.wt“ in Bln 94)“" und ,nk.wt" (Bln 35) für „nk.wt" (Eb 122) ,geritzte Sykomorenfrüchte“ gebildet. ${ }^{4}$

Der hier vorgelegte Beitrag wird im Wesentlichen zwei Ziele verfolgen. Die Reihe soll auf der einen Seite um neue Beispiele für den Wechsel zwischen "“ und „, $k^{*}$ erweitert werden. Die Untersuchung wird auf der anderen Seite auch die beiden anderen Gutturale , $k^{\prime \prime}$ und,$g^{\prime \prime}$ mit einbeziehen. Die beiden letzten Konsonanten sollen dabei ebenfalls auf die Möglichkeit eines Austausches mit "“ überprüft werden. Die Informationen sollen dazu auf möglichst nachvollziehbare Art und Weise gebündelt werden. Der weitere Verlauf wird zeigen, dass das Lautgesetz für alle drei Gutturale gegolten hat. Die Austauschbarkeit ${ }^{5}$ der Gutturale untereinander wird hierbei ein Übriges getan haben. Das Alter der Belege geht aus den Angaben in den Klammern hervor. Die frühesten Beispiele datieren noch ins Mittlere Reich, während sich die Hauptmasse der Belege auf das Neue Reich verteilt. Der - summarische - Querverweis auf noch unveröffentlichte Arbeiten des Autors $\mathrm{zu}$ anderen Lautwandeln hat sich leider häufig nicht vermeiden lassen, weil die komplette Aufzählung jedes einzelnen Beispiels die Fußnoten hoffnungslos überfrachtet hätte. Der Leser wird dafür um Verständnis gebeten.

WESTENDORF, Grammatik der medizinischen Texte, Grundriss der Medizin der alten Ägypter VIII (Berlin: Akademie-Verlag, 1962), 19.

${ }^{4}$ W. WESTENDORF, Grammatik der medizinischen Texte, Grundriss der Medizin der alten Ägypter VIII (Berlin: Akademie-Verlag, 1962), 18. Zum Ausfall von „““ allgemein vgl. C. PEUST, Egyptian Phonology, An Introduction to the Phonology of a dead Language (Göttingen: Peust \& Gutschmidt Verlag, 1999), 102; zur Schwäche des "“ in anderen Sprachen vgl. R. C. STEINER, Early Northwest Semitic Serpent Spells in the Pyramid Texts, Harvard Semitic Studies 61 (Winona Lake: Eisenbrauns, 2011), 68 n. 45.

${ }^{5}$ W. WESTENDORF, Grammatik der medizinischen Texte, Grundriss der Medizin der alten Ägypter VIII (Berlin: Akademie-Verlag, 1962), 43. 


\section{Der Austausch zwischen , "“ und ,,$k^{*}$}

In Hinblick auf den Austausch zwischen „“" und „, $k^{\text {“ }}$ können der Liste noch drei neue Beispiele hinzugefügt werden.

Das erste Beispiel ist das Wortspiel zwischen „ک“" „Leiter“ und

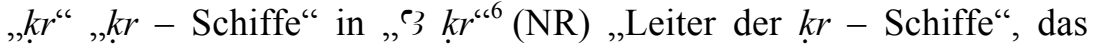
durchaus mit dem Austausch zwischen ," und , $k^{\prime \prime}$ in Verbindung gebracht werden kann. Das Wortspiel ist außerdem auf Grundlage des Austausches zwischen , $3^{\text {“ }}$ und ,,$r^{6 / 7}$ zustande gekommen.

Das zweite Beispiel besteht aus dem Wortspiel zwischen , $\mathrm{km} 3^{\text {“ }}$

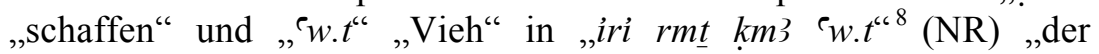
(=Amun-Re) die Menschen machte und das Vieh erschuf" sowie „ib3 $n=k{ }^{\prime}$ w.t $k m 3 . n=k^{69} /, i b 3 \quad n=k$ ' w.t $k m 3<t>. n=k^{* 10}$ (NR) ,das Vieh tanzt für dich (=Amun-Re), das du geschaffen hast“/,die Wildtiere, die du geschaffen hast, tanzen vor dir (=Amun-Re)“, das allerdings nur unter den nötigen Vorbehalten gegeben wird. Die lautlichen Ursachen für dieses Wortspiel könnten aber schnell benannt werden. Das Wortspiel hätte im Falle seines Bestehens nicht nur den Austausch zwischen "“" und , $k^{*}$, sondern auch den Austausch zwischen , $m^{\prime \prime}$ und , $w^{\text {c11 }}$ sowie ,"“" und , , $w^{\text {c12 }}$ beinhaltet.

${ }^{6}$ J. OSING, Das Grab des Nefersecheru in Zawyet Sultan, Archäologische Veröffentlichungen 88 (Mainz: von Zabern, 1992), 57. Zum „kr" - Schiff vgl. N. DÜRRING, Materialien zum Schiffbau im alten Ägypten, Abhandlungen des Deutschen Archäologischen Instituts Kairo, Ägyptologische Reihe, Band 11 (Berlin: Achet Verlag, 1995), 141.

${ }^{7}$ K. SETHE, Das aegyptische Verbum im Altaegyptischen, Neuaegyptischen und Koptischen, Erster Band, Laut- und Stammeslehre (Leipzig: J. C. Hinrichs'sche Buchhandlung, 1899), 50; W. WESTENDORF, Grammatik der medizinischen Texte, Grundriss der Medizin der alten Ägypter VIII (Berlin: Akademie-Verlag, 1962), 32.

${ }^{8}$ M. M. LUISELLI, Der Amun-Re Hymnus des P. Boulaq 17 (P. Kairo CG 58038), Kleine Ägyptische Texte 14 (Wiesbaden: Harrossowitz, 2004), 2/25.

${ }^{9}$ J. ASSMANN, Sonnenhymnen in thebanischen Gräbern, Theben 1 (Mainz: von Zabern, 1983), 27.

${ }^{10}$ J. ASSMANN, Sonnenhymnen in thebanischen Gräbern, Theben 1 (Mainz: von Zabern, 1983), 363.

${ }^{11}$ K. SETHE, Das aegyptische Verbum im Altaegyptischen, Neuaegyptischen und Koptischen, Erster Band, Laut- und Stammeslehre (Leipzig: J. C. Hinrichs'sche Buchhandlung, 1899), 126/127; W. WESTENDORF, Grammatik der medizinischen Texte, Grundriss der Medizin der alten Ägypter VIII (Berlin: Akademie-Verlag, 1962), 26.

${ }^{12}$ W. WESTENDORF, Grammatik der medizinischen Texte, Grundriss der Medizin der alten Ägypter VIII (Berlin: Akademie-Verlag, 1962), 11. 
Die Schreibung , $k h^{“ 13}$ für ,,$k^{\complement} h^{“}$ „Ecke, Winkel, Seite“ deutet möglicherweise ebenfalls auf den Austausch zwischen „" " und , $k^{\prime \prime}$ hin. Die Assimilation von „" "an , $k^{\prime \prime}$ kann zumindest als eine denkbare Option verstanden werden. Die Erklärung mit der Assimilation an,$h^{\prime “}$ aufgrund des Austausches zwischen „“ und „, $h^{\text {“14 }}$ ist allerdings ebenfalls nicht gänzlich von der Hand zu weisen.

\section{Der Austausch zwischen ," "“ und ,,$k^{6 "}$}

In den nächsten Zeilen wird es thematisch um den Austausch zwischen "“" und „,k“ gehen. Die hierfür in Frage kommenden

13 H.-W. FISCHER-ELFERT, Literarische Ostraka der Ramessidenzeit in Übersetzung, Kleine Ägyptische Texte 9 (Wiesbaden: Harrassowitz, 1986), 9 a.

${ }^{14}$ J. OSING, Der spätägyptische Papyrus BM 10808, Ägyptologische Abhandlungen 33 (Wiesbaden: Harrassowitz, 1976), 236; J. OSING, Die Nominalbildung des Ägyptischen, Anmerkungen und Indices, Deutsches Archäologisches Institut Abteilung Kairo, Sonderschrift 3b (Mainz: von Zabern, 1976), 509; W. WESTENDORF, Pyramiden und Sonnenbahn, in: Festschrift Arne Eggebrecht zum 65. Geburtstag am 12. März 2000, Hildesheimer Ägyptologische Beiträge 48 (Hildesheim: Gerstenberg Verlag, 2002), 134; W. WESTENDORF, Grammatik der medizinischen Texte, Grundriss der Medizin der alten Ägypter VIII (Berlin: Akademie-Verlag, 1962), 36; C. PEUST, Egyptian Phonology, An Introduction to the Phonology of a dead Language, Monographien zur ägyptischen Sprache Band 2 (Göttingen: Peust \& Gutschmidt Verlag, 1999), 105/194. Zum Verhältnis zwischen "“" und , $h$ "vgl. auch E. EDEL, Altägyptische Grammatik, Analecta Orientalia 34/39 (Rom: Pontificium Institutum Biblicum, 1955/1964), 54. Der Lautwandel könnte auch die Assimilation von „“" an , $h$ “ in den Wortspielen zwischen , $b h^{\Upsilon}$ “ ,überfluten“ und „hbl „Fest“ bei M. SANDMAN, Texts from the time of Akhenaten, Bibliotheca Aegyptiaca 8 (Bruxelles, 1938), 91, sowie „b`h“ „,überfluten“ und , $h b b(. t)$ “ „frisches Wasser" bei D. KURTH, Edfou VII, Die Inschriften des Tempels von Edfu, Abteilung I, Übersetzungen, Band 2 (Wiesbaden: Harrassowitz, 2004), 213, in Gang gebracht

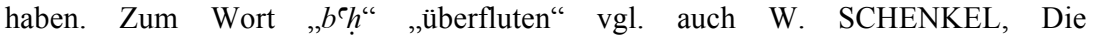
Bewässerungsrevolution im Alten Ägypten, Deutsches Archäologisches Institut Abteilung Kairo, Sonderschrift 6 (Mainz: von Zabern, 1978), 25f; G. TAKÁCS, Etymological Dictionary of Egyptian, Volume One: A phonological Introduction, Handbuch der Orientalistik, Erste Abteilung, Der Nahe und Mittlere Osten, Achtundvierzigster Band (Leiden/Boston/Köln: Brill, 1999), 330. Zum Wortspiel

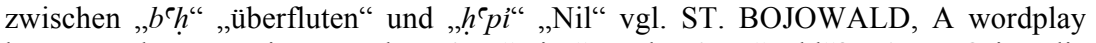
between the Egyptian words wbn "arise" and $n b w$ "gold"?, Acta Orientalia Academiae Scientiarum Hungaricae 64 (3) (2011), 358f. 


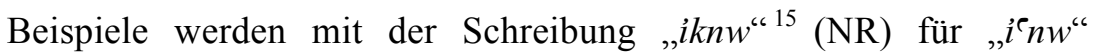
„Kummer“ begonnen. Die Schreibung würde durch den Austausch zwischen „," und „, $k$ " eine hinreichende Erklärung finden.

Der Austausch zwischen "“ und „, $k^{\prime \prime}$ hat auch die lautliche Voraussetzung für das Wortspiel zwischen „, ${ }^{\text {‘“ }}$,groß“ und „ikn“ , $i k n^{16}$ - Krug“ in , ikn 93 m b3k h3rww"17 (NR) ,großer $i k n$ - Krug als

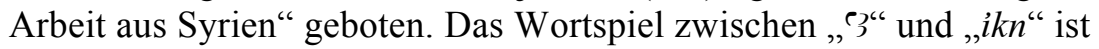
jedoch nicht allein auf den Austausch zwischen " " und , $k^{\text {" }}$ zurückzuführen. In seinem Fall ist vielmehr das Zusammenspiel noch weiterer Faktoren zu beobachten, unter denen der Austausch zwischen

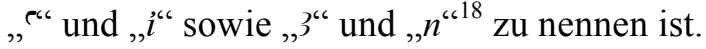

Das Wortspiel zwischen „k $k$ “" „Stier“ und , ${ }^{3} 3$ “" befruchten“ in

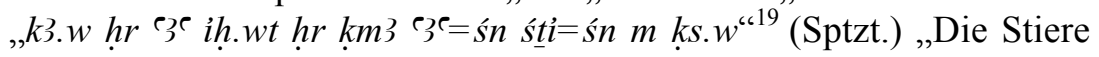
begatten die Kühe, während sie den Samen erschaffen, den sie von Knochen ejakulieren “20 sollte ebenfalls in gebührender Weise zur Kenntnis genommen werden. Das Wortspiel könnte sogar noch weiter auf die , ih. $t^{\text {" }}$ - Kuh ausgedehnt werden, wozu nur der Austausch zwischen "“" und ,,$"$ " sowie , "“ und , $h$ " angenommen werden muss. Der Austausch zwischen „,“ und ,h" $h^{\circ}$ wird an anderer Stelle eine eigene Untersuchung erhalten.

\footnotetext{
${ }^{15}$ I. MUNRO, Das Totenbuch des Pa-en-nesti-taui aus der Regierungszeit des Amenemope (pLondon BM 10064), Handschriften des Altägyptischen Totenbuches 7 (Wiesbaden: Harrassowitz, 2001), 13.

${ }^{16}$ Zum ,ikn“ - Krug vgl. FR. CALICE, Grundlagen der ägyptisch-semitischen Wortvergleichung, Eine kritische Diskussion des bisherigen Vergleichsmaterials, Beihefte zur „Wiener Zeitschrift für die Kunde des Morgenlandes“, 1. Heft (Wien: Selbstverlag des Orientalischen Institutes der Universität Wien, 1936), 123; TH. O. LAMBDIN, Egyptian Words in Tell el Amarna Letter No. 14, Orientalia 22 (1953), 363; J. OSING, Die Nominalbildung des Ägyptischen, Anmerkungen und Indices, Deutsches Archäologisches Institut Abteilung Kairo, Sonderschrift 3B (Mainz: von Zabern, 1976), 733f; Z. COCHAVI-RAINEY, Egyptian Influence in the Amarna Texts, Ugarit - Forschungen 29 (1997), 95-96.

${ }^{17}$ Urk. IV, 665, 16.

${ }^{18}$ W. WESTENDORF, Grammatik der medizinischen Texte, Grundriss der Medizin der alten Ägypter VIII (Berlin: Akademie-Verlag, 1962), 22.

${ }^{19}$ D. KLOTZ, Adoration of the Ram, Five Hymns to Amun-Re from Hibis Temple, Yale Egyptological Studies 6 (New Haven, 2006), 149.

${ }^{20}$ Andere Auffassung der Stelle bei C. KNIGGE, Das Lob der Schöpfung, Die Entwicklung ägyptischer Sonnen- und Schöpfungshymnen nach dem Neuen Reich, Orbis Biblicus et Orientalis 219 (Fribourg: Academic Press/Göttingen: Vandenhoeck \& Ruprecht, 2006), 266.
} 


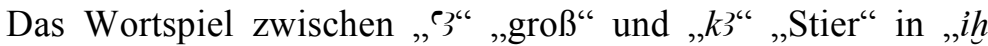
$w h 3=k 4$ k3.w nfr.w sp śn.nw 3.w sp śn.n $w^{\text {“621 }}$ (NR) ,Seek out 4 bulls, very fine and very large ${ }^{\text {‘22 }}$ ist möglicherweise ebenfalls als Folge des Austausches zwischen , $k$ " und „" zustande gekommen. Die Neigung

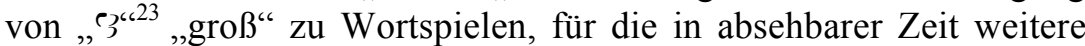
Beispiele vorgestellt werden sollen, ist bereits an den wenigen bisher genannten Beispielen zu sehen.

Der Austausch zwischen „" " und „ $k^{\text {" }}$ tritt auch im Wortspiel

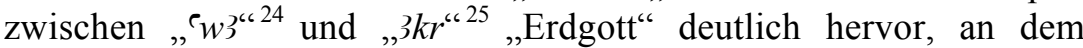
außerdem der Austausch zwischen , $r^{\prime \prime}$ und , $w^{\prime \prime}$ beteiligt war. Der Austausch zwischen , $w^{\prime \prime}$ und „, $r^{\prime \prime}$ ist zwar von Westendorf ${ }^{26}$ als zweifelhaft bezeichnet worden. Wie jedoch an anderer Stelle gezeigt werden soll, scheint es gute Gründe für seine Existenz zu geben. Das Wortspiel zwischen " "w $\}^{\prime \prime}$ und ,,$k r^{\prime \prime}$ ist außerdem auf Basis einer Metathese entstanden.

${ }^{21}$ A. H. GARDINER, Late-Egyptian Miscellanies, Bibliotheca Aegyptiaca VII (Bruxelles, 1937), 80

${ }^{22}$ R. A. CAMINOS, Late-Egyptian Miscellanies, Brown Egyptological Studies I (Oxford: University Press, 1954), 305.

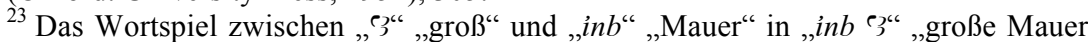
(von Theben)" bei L. GOLDBRUNNER, Buchis, Eine Untersuchung zur Theologie des heiligen Stieres in Theben zur griechisch-römischen Zeit, Monographiès Reine Élisabeth 11 (Turnhout: Brepols, 2004), 56, stellt sich dabei als mögliche

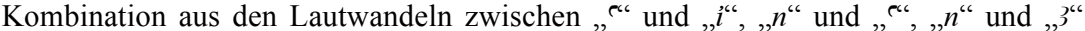

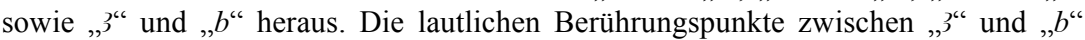
sollen in anderem Zusammenhang herausgestellt werden. Zum Wort ,inb“ „Mauer“ und seinem arabischen Etymon vgl. FR. CALICE, Zur ägyptisch-semitischen Wurzelverwandtschaft, Zeitschrift für ägyptische Sprache und Altertumskunde 39 (1901), 146.

24 B. LÜSCHER, Totenbuch Spruch 149/150, Totenbuchtexte, Synoptische Textausgabe nach Quellen des Neuen Reiches, Band 6 (Basel: Orientverlag, 2010), 70.

${ }^{25}$ Zum Wort „3kr” „Erdgott“ vgl. J. FR. QUACK, Zur Stellung des Ägyptischen innerhalb der afroasiatischen Sprache, Orientalistische Literaturzeitung 97 (2002) 2, 168.

${ }^{26}$ W. WESTENDORF, Grammatik der medizinischen Texte, Grundriss der Medizin der alten Ägypter VIII (Berlin: Akademie-Verlag, 1962), 22. 


\section{Der Austausch zwischen "ृ“" und ,g“}

Die nächsten Beispiele werden den Austausch zwischen „"“ und , ${ }^{\text {“ }}$

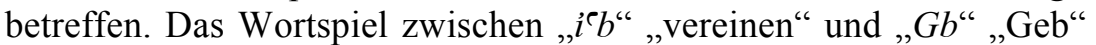
in der Verbindung , „ic $b=k k s s . w=k h r G b^{، 27}$ (NR) ,Vereinige deine Knochen bei Geb" wird hier an den Anfang gestellt, bei dem die Erklärung mit dem Austausch zwischen "“" und "g" zumindest vorsichtig in Erwägung gezogen werden kann. Die Möglichkeit ist allerdings nicht ausgeschlossen, dass in diesem Fall auch der Austausch zwischen , $g^{\prime \prime}$ und , $i$ “ eine gewisse Rolle gespielt hat. Die Beispiele für den Austausch zwischen , „“" und Gutturalen sollen in einer eigenen Untersuchung behandelt werden. In der Zwischenzeit mag der Hinweis auf die Bemerkungen bei Klotz ${ }^{28}$ genügen.

Das Wortspiel zwischen „gmi" „finden“ und „ک3m“ „Bauer“ in „bn-p=i gmi $3 m$....29 (Sptzt.) „Ich habe keinen Bauern gefunden...“ wird hier an zweiter Stelle genannt, für das der Austausch zwischen „“" und „g“ eine durchaus schlüssige Erklärung sein könnte.

Die gleiche Deutung trifft auf das Wortspiel zwischen ,gmi“

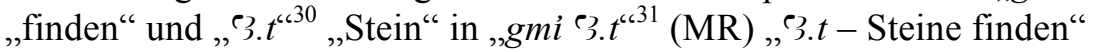
$\mathrm{zu}$, das sowohl mit dem Austausch zwischen ,g“" und ," als auch dem

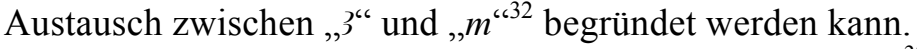

Das Wortspiel zwischen „gmi“" „finden“" und „, „3“333 „Esel“" in „gmi c $^{\text {“34 }}$ (Sptzt.) „Esel finden“ ist auf Grundlage der gleichen

${ }^{27}$ M. SANDMAN, Texts from the time of Akhenaten, Bibliotheca Aegyptiaca VIII (Bruxelles, 1938), 140.

${ }^{28}$ D. KLOTZ, Adoration of the Ram, Five Hymns to Amun-Re from Hibis Temple, Yale Egyptological Studies 6 (New Haven, 2006), 121 n. 359.

${ }^{29}$ W. SPIEGELBERG, Demotica I, Sitzungsberichte der Bayerischen Akademie der Wissenschaften, Philosophisch-philologische und historische Klasse, Jahrgang 1925, 6. Abhandlung (München: Verlag der Bayerischen Akademie der Wissenschaften in Kommission des G. Franz'schen Verlags (J. Roth), 1925), 21.

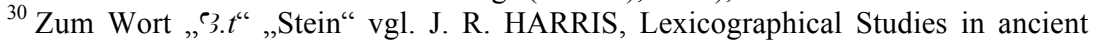
Egyptian Minerals, Deutsche Akademie der Wissenschaften zu Berlin, Institut für Orientforschung, Veröffentlichungen Nr. 54 (Berlin: Akademie-Verlag, 1961), $21 \mathrm{f}$.

${ }^{31}$ MM. J. COUYAT/P. MONTET, Les inscriptions Hiéroglyphiques et Hiératiques du Ouadi Hammamat, Mémoires publiés par les members de 1'Institut Francais d' Archéologie Orientale du Caire, Tome Trente - Quatrième (Le Caire, 1912), 102.

${ }^{32} \mathrm{~W}$. WESTENDORF, Grammatik der medizinischen Texte, Grundriss der Medizin der alten Ägypter VIII (Berlin: Akademie-Verlag, 1962), 11.

${ }^{33}$ Zum Wort "3" „Esel“" vgl. W. VYCICHL, Grundlagen der ägyptisch-semitischen Wortvergleichung, Mitteilungen des Deutschen Archäologischen Instituts Abteilung 
Modalitäten gebildet, so dass die Erklärung aus dem letzten Fall unverändert übernommen werden kann.

Der Austausch zwischen „" und „g“ zeigt sich außerdem im Wortspiel zwischen dem Wort (?) mit Determinativ des „Speichelnden Mundes“ und „pśg“ „,bespeien“ in ,p $p^{\tau} . t h r$ pśg $g^{\text {‘.35 }}$ (NR),

Kairo 16 (1958), 380; FR. CALICE, Grundlagen der ägyptisch-semitischen Wortvergleichung, Eine kritische Diskussion des bisherigen Vergleichsmaterials, Beihefte zur „Wiener Zeitschrift für die Kunde des Morgenlandes“, 1. Heft (Wien: Selbstverlag des Orientalischen Institutes der Universität Wien, 1936), 25; P. M. LACAU, Études d'Égyptologie, I. Phonétique Égyptienne Ancienne, Bibliothèque d' Étude 41 (Le Caire, 1970), 13; E. EDEL, Beiträge zum ägyptischen Lexikon II., Zeitschrift für Ägyptische Sprache und Altertumskunde 81 (1956), 9-11; G. TAKÁCS, Etymological Dictionary of Egyptian, Volume One: A Phonological Introduction, Handbuch der Orientalistik, Erste Abteilung, Der Nahe und Mittlere Osten, Achtundvierzigster Band (Leiden/Boston/Köln: Brill, 1999), 54; R. C. STEINER, Early Northwest Semitic Serpent Spells in the Pyramid Texts, Harvard Semitic Studies 61 (Winona Lake: Eisenbrauns, 2011), 65. Zur Rolle des Esels in der Handwerkersiedlung von Deir el-Medina vgl. J. J. JANSSEN, Donkeys at Deir elMedina, Egyptologische Uitgaven XIX (Leiden, 2005), VII-XI, 2-127. Beachte das

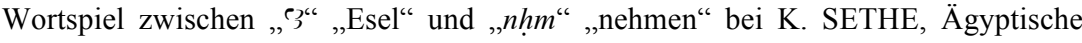
Lesestücke zum Gebrauch im akademischen Unterricht, Texte des Mittleren Reiches, Dritte unveränderte Auflage (Hildesheim: Georg Ohms Verlagsbuchhandlung Hildesheim, 1959), 19; H. GOEDICKE/E. F. WENTE, Ostraka Michaelides (Wiesbaden: Harrassowitz, 1962), Nr. 2, das neben dem Lautwandel zwischen „“" und , $n$ “ auch die Lautwandel zwischen ," und , $h$ " sowie ,"“ und , $m$ " einschließt. Zum Lautwandel zwischen "“" und „," vgl. W. WESTENDORF, Bemerkungen und Korrekturen zum Lexikon der Ägyptologie (Göttingen: Hubert \& Co, 1989), 109,

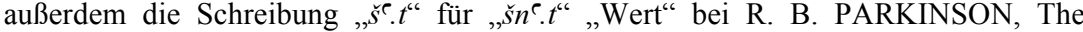
discourse of the fowler: Papyrus Butler Verso (P. BM EA 10274), The Journal of Egyptian Archaeology 90 (2004), 108/110, sowie das Wortspiel zwischen , „3“" „ejakulieren“ und „nwn“ „Nun“ bei D. KLOTZ, Adoration of the Ram, Five Hymns to Amun-Re from Hibis Temple, Yale Egyptological Studies 6 (New Haven, 2006), 21.

${ }^{34}$ W. ERICHSEN, Eine ägyptische Schulübung in demotischer Schrift, Det Kgl. Danske Videnskabernes Selskab, Historisk-Filologiske Meddelelser, Bind XXXI, Nr. 4 (Kobenhavn, I Kommission hos Ejnar Munksgaard, 1948), 9; FR. HOFFMANN, Der literarische demotische Papyrus Wien D 6920-22, Studien zur altägyptischen Kultur 23 (1996), 177.

${ }^{35}$ A. GASSE, Catalogue des ostraca Hiératiques littéraires de Deir el-Médina, $\mathrm{N}^{\text {os }}$ 1676-1774, Tome IV, Fasc. 1, Documents de Fouilles, T. XXV (Le Caire, 1990), 1684 (x + 5). Zum Wort „pśg“ „,speien“ vgl. FR. BEHNK, Lexikalische Beiträge zur ägyptisch-semitischen Sprachvergleichung, Zeitschrift für ägyptische Sprache und Altertumskunde 62 (1967/Neudruck der Ausgabe 1927), 81. 
der hier mit dem Austausch zwischen „““ und , „S ${ }^{\star 36}$ vermengt worden ist. In diesem Zusammenhang kann als Parallele auf die Schreibung

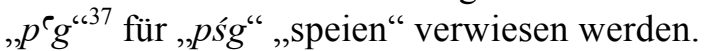

Die Metathese , $n g^{\text {c‘38 }}$ (NR) für das ältere , $n^{\complement} g^{\text {“ }}$, ,zerkleinern“ könnte ein weiteres Indiz für den Austausch zwischen ", " und , ," bilden.

Die Schreibungen $\square \mathbb{A}=\mathbb{R} \mathbb{1}^{39}$ (NR) als Tätigkeitsverb der ,śmn“ - Gans können unter Umständen als weitere Beispiele für den Austausch zwischen "“" und "g" interpretiert werden. Die Synopse bei Lapp macht deutlich, dass die Wörter in der einen Textversion für die Wörter „,ng/ngg“ „schnattern“ in den anderen Textversionen eingesprungen sind. Das in die Schreibung hineingeschmuggelte "“ könnte durchaus mit dem Austausch zwischen "“" und "g" begründet werden. Die Erklärung mit dem Austausch zwischen "“ und "g“ stellt allerdings nicht die einzig mögliche Alternative dar. In gleicher Weise könnte der oben ge-

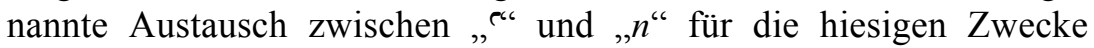
instrumentalisiert werden. Die Formen müssten unabhängig von der endgültigen Entscheidung als unetymologische Schreibungen bezeichnet werden. Das zusätzliche ,““ könnte mit der von Westendorf ${ }^{40}$ besprochenen Einfügung eines bedeutungslosen ,““ erklärt werden. Das gleiche Prinzip ist bei den Schreibungen , $n g 3 g 3^{3441}$

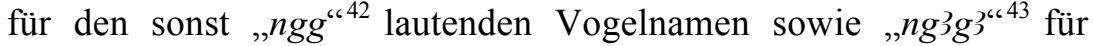

${ }^{36}$ W. WESTENDORF, Bemerkungen und Korrekturen zum Lexikon der Ägyptologie (Göttingen: Hubert \& Co, 1989), 19.

${ }^{37}$ WB I, 504, 15.

${ }^{38}$ WB II, 349, 13; N. TACKE, Verspunkte als Gliederungsmittel in ramessischen Schülerhandschriften, Studien zur Archäologie und Geschichte Altägyptens, Band 22 (Heidelberg: Heidelberger Orientverlag, 2001), 114.

${ }^{39}$ G. LAPP, Totenbuch Spruch 17, Totenbuchtexte, Synoptische Textausgabe nach Quellen des Neuen Reiches, Band 1 (Basel: Orientverlag, 2006), 244.

${ }^{40} \mathrm{~W}$. WESTENDORF, Grammatik der medizinischen Texte, Grundriss der Medizin der alten Ägypter VIII (Berlin: Akademie-Verlag, 1962), 9. Die Hinzufügung von „з“ bei K. SETHE, Das aegyptische Verbum im Altaegyptischen, Neuaegyptischen und Koptischen, Erster Band, Laut- und Stammeslehre (Leipzig: J. C. Hinrichs'sche Buchhandlung, 1899), 44, ist hingegen als phonetisches Komplement zu verstehen.

${ }^{41}$ CHR. LEITZ, Magical and Medical Papyri of the New Kingdom, Hieratic Papyri in the British Museum VII (London: British Museum Press, 1999), 41 n. 80. Beachte die Schreibung ,ng3g;““ für ,nh3h 3““ ,strotzen“ bei E. EDEL, Altägyptische Grammatik, Analecta Orientalia 34/39 (Rom: Pontificium Institutum Biblicum, 1955/1964), 53.

${ }^{42}$ Beachte die Nebenform ,gg“ zum „ngg“ - Vogel bei WB II, 350. 
„ngg“ „schnattern“ festzustellen. Die Erklärung des Wortes „ng3g3““ als Nebenform für „ngngn“ „,schnattern“ bei Grapow ${ }^{44}$ trifft den Kern der Sache nur halb, da ",ngngn“ seinerseits eine Nebenform für „,ngg“" sein dürfte. Die Form „,ngngn“" ist dem Wörterbuch ${ }^{45}$ zwar nicht für „ngg“ „schnattern“, aber dafür beim „,ngg“ - Vogel bekannt.

Der Austausch zwischen "," und „g" spiegelt sich vielleicht

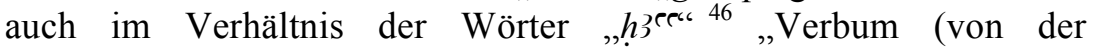
Schifffahrt)“ und , $h 3 g^{6447}$ „Verbum von der Schifffahrt“ (MR) wider. Die Nähe der beiden Wörter ist bereits von Vogelsang ${ }^{48}$ erkannt worden, dem ,h $3^{\text {rec }}$ höchst verdächtig vorgekommen war. Das Problem hat Westendorf ${ }^{49}$ dadurch zu beheben versucht, dass er dem

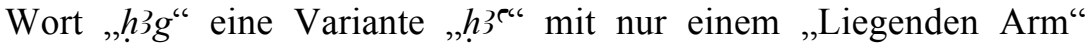
gegenübergestellt hat. Die These beruht auf der Annahme, dass der ursprüngliche zweite „Liegende Arm“ von , $h 3^{\text {rec }}$ in das Determinativ des „Schlagenden Armes“ zu emendieren ist. Der Austausch zwischen "“ und ,g" war zwar Westendorf nach eigenen Angaben noch nicht begegnet. Die Schreibungen hat er aber vor dem Hintergrund der Tatsache erklären wollen, dass semitisches „“ im Ägyptischen stellenweise in palatalisiertes,$g^{\prime \prime}$ oder aber,$h$ “ übergegangen ist. Im letzten Fall wäre zusätzlich der ägyptische Wechsel zwischen „, $h^{\text {“ und }}$ "g“ zu berücksichtigen. Der Austausch zwischen , " und ,g" hat aber durch die hier präsentierten Beispiele einen etwas größeren Grad an Wahrscheinlichkeit gewonnen. Der Umweg über das Semitische würde auf diese Weise unnötig.

Die Erwähnung der Schreibungen , $n^{\Upsilon} g / n^{`} g g^{\prime \prime}$ für ,,ng/ngg“" lädt abschließend zu einem Exkurs ein, in dem gezeigt werden soll, dass ein scheinbar überflüssiges „“ auch in die Schreibung anderer Wörter

\footnotetext{
${ }^{43}$ WB II, 350, Belegschreibungen.

${ }^{44}$ H. GRAPOW, Beiträge zur Erklärung des Totenbuches, Zeitschrift für ägyptische Sprache und Altertumskunde 49 (1967/Neudruck der Ausgabe 1911), 45.

${ }^{45}$ WB II, 350, Belegschreibungen.

${ }^{46}$ WB IV, 30, 4.

${ }^{47}$ WB IV $, 35,1$.

${ }^{48}$ FR. VOGELSANG, Kommentar zu den Klagen des Bauern, Untersuchungen zur Geschichte und Altertumskunde Aegyptens VI (Leipzig: J. C. Hinrichs'sche Buchhandlung, 1913), 69.

${ }^{49}$ W. WESTENDORF, Das strandende Schiff, Zur Lesung und Übersetzung von Bauer B 1, 58 = R 101, in: Jan Assmann/Erika Feucht/Reinhard Grieshammer (Hrsg.), Fragen an die altägyptische Literatur, Studien zum Gedenken an Eberhard Otto (Wiesbaden: Dr. Ludwig Reichert Verlag, 1977), 507.
} 
hineintransportiert worden ist. Das Phänomen ist im Prinzip nicht neu, da der Zusatz eines offenbar bedeutungslosen " "für das römische Demotisch bereits von Peust ${ }^{50}$ besprochen worden ist. Im Anschluss soll zunächst ein Beispiel nachgetragen werden, das Peust zu seiner Zeit noch nicht kennen konnte. Das neue Beispiel ist in der

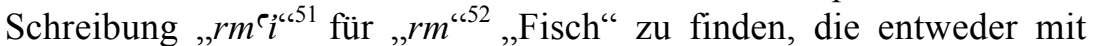

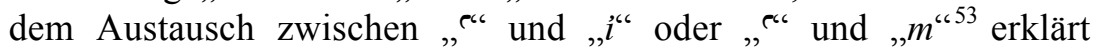
werden kann. Das Alter der nach bisheriger Meinung ganz späten Erscheinung muss aber nach dem oben zitierten Beispiel aus dem Neuen Reich wesentlich höher angesetzt werden. Der Eindruck wird sich durch die folgenden Beispiele noch verstärken. Die Schreibung

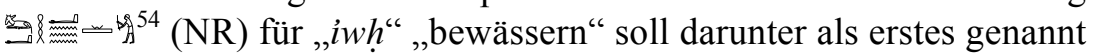
werden, die von dieser Gesetzmäßigkeit gleich doppelt betroffen war. Was zunächst das "“" unterhalb des liegenden Antilopenkälbchens angeht, so könnte es durch den Lautwandel zwischen „“" und „h" $h^{\prime \prime}$ oder " " und „w" in die Schreibung gelangt sein. Die Hauptursache für das "“ über den „Liegenden Wasserlinien“ hingegen ist laut Fischer-

\footnotetext{
${ }^{50}$ C. PEUST, Egyptian Phonology, An Introduction to the Phonology of a dead Language, Monographien zur ägyptischen Sprache Band 2 (Göttingen: Peust \& Gutschmidt Verlag,1999), 102.

${ }^{51}$ B. MUHS/J. DIELEMAN, A bilingual account from late Ptolemaic Tebtunis, Zeitschrift für ägyptische Sprache und Altertumskunde 133 (2006), 60 n. i.

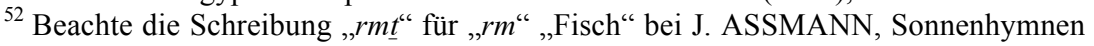
in thebanischen Gräbern, Theben 1 (Mainz: von Zabern, 1983), 282. Die Bedeutung „Fisch“ von „rm“ konnte auch zu „Wassertier“ verallgemeinert werden, vgl. H. BRUNNER, Eine Dankstele an Upuaut, Mitteilungen des Deutschen Archäologischen Institutes Abteilung Kairo 16, Festschrift zum 80. Geburtstag von Professor Dr. Hermann Junker, II. Teil (1958) 7; E. HORNUNG, Das Amduat, Die Schrift des verborgenen Raumes, Herausgegeben nach Texten aus den Gräbern des Neuen Reiches, Teil II: Übersetzung und Kommentar, Ägyptologische Abhandlungen 7, 2 (Wiesbaden: Harrassowitz, 1963), 38. Das Wort „rm“ „Fisch“ war vielleicht auch für das Fischdeterminativ nach dem Wort , $r^{c} m^{\prime \prime}$ bei A. H. GARDINER, Ancient Egyptian Onomastica, Text, Volume II (Oxford: University Press, 1947), 253*, zuständig, dessen Bedeutung allerdings unklar ist. Das gleiche Wort könnte aber bei I. E. S. EDWARDS, Hieratic Papyri in the British Museum, Fourth Series, Oracular Amuletic Decrees of the Late New Kingdom, Vol. I, Text (London, 1960), 70 n. 26, vorliegen. Die einzige Sicherheit hat offenbar darin bestanden, dass es sich bei , $r^{c} m^{\text {“ }}$ um ein inneres Organ handeln könnte.

${ }^{53}$ W. WESTENDORF, Grammatik der medizinischen Texte, Grundriss der Medizin der alten Ägypter VIII (Berlin: Akademie-Verlag, 1962), 26.

54 H.-W. FISCHER-ELFERT, Literarische Ostraka der Ramessidenzeit in Übersetzung, Kleine Ägyptische Texte 9 (Wiesbaden: Harrassowitz, 1986), 40.
} 


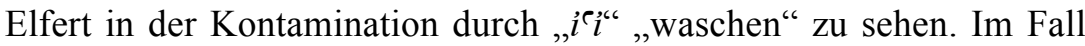
der Richtigkeit dieses Ansatzes würde die Verschreibung zwischen , $i^{\circ *}$ und,$h^{\star 65}$ besonderes Gewicht erhalten. Das Wörterbuch ${ }^{56}$ dagegen hat hier fragend eine seit der 19. Dynastie belegte Nebenform, iw $h^{\text {e“ }}$ $\mathrm{zu}, i w h^{\prime \prime}$ angenommen. Die Schreibung $2\left(\mathrm{~N}-\beta\right.$ (NR) für die , $m h . t^{*}$ Feder ist als nächstes Beispiel zu erwähnen, bei der das zusätzliche „““ mit der Kontamination durch „mhei“ „Flachs“ zu erklären sein dürfte ${ }^{57}$. Die Schreibung, $f^{\top 3} . t^{658}$ (Sptzt.) setzt den Schlusspunkt unter dieses Kapitel, die ebenfalls zu diesem Komplex gehören könnte. In ihrem Fall hat es sich offenbar um eine Schreibung für ,f fiil.t. ${ }^{659}$ „Kanal“" gehandelt.

\footnotetext{
${ }^{55} \mathrm{Zu}$ dieser Verschreibung vgl. E. EDEL, Altägyptische Grammatik, Analecta Orientalia 34/39 (Rom: Pontificium Institutum Biblicum, 1955/1964), 263.

${ }^{56}$ WB I, 57, Vorbemerkung zu ,iwh".

${ }^{57}$ M. RÖMER, Der Kairener Hymnus an Amun-Re, Zur Gliederung von pBoulaq 17, in: Jürgen Osing/Günter Dreyer (Hrsg.), Form und Maß, Beiträge zur Literatur, Sprache und Kunst des alten Ägypten, Festschrift für Gerhard Fecht zum 65. Geburtstag am 6. Februar 1987, Ägypten und Altes Testament 12 (Wiesbaden: Harrassowitz, 1987), 423.

${ }^{58}$ J. FR. QUACK, Rezension zu: Takács, Gábor: Etymological Dictionary of Egyptian. Vol. 2: $b-, p-, f-$. Leiden, Boston, Köln: Brill 2001. Handbuch der Orientalistik, Abt. 1: Der Nahe und Mittlere Osten, 48, Orientalistische Literaturzeitung 99 (2004) 1, 41.

${ }^{59}$ Zum Wort ,f 3ii.t. „Kanal“" vgl. auch J. OSING, Die Partizipien im Ägyptischen und in den semitischen Sprachen, in: Jürgen Osing/Günter Dreyer (Hrsg.), Form und Maß, Beiträge zur Literatur, Sprache und Kunst des alten Ägypten, Festschrift für Gerhard Fecht zum 65. Geburtstag am 6. Februar 1987, Ägypten und Altes Testament 12

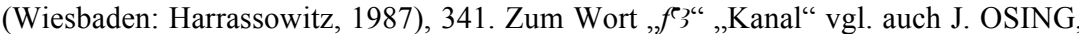
Die Nominalbildung des Ägyptischen, Anmerkungen und Indices, Deutsches Archäologisches Institut Abteilung Kairo, Sonderschrift 3B (Mainz: von Zabern, 1976), 599.
} 
